\title{
Tableau des chaires depuis 1800
}

\section{(2) OpenEdition}

1 Journals

Édition électronique

URL : https://journals.openedition.org/annuaire-cdf/1905

DOI : 10.4000/annuaire-cdf. 1905

ISBN : 978-2-7226-0325-7

ISSN : 2109-9227

Éditeur

Collège de France

Édition imprimée

Date de publication : 1 avril 2013

Pagination : 23-57

ISBN : 978-2-7226-0198-7

ISSN : 0069-5580

\section{Référence électronique}

"Tableau des chaires depuis 1800 », L'annuaire du Collège de France [En ligne], 112 | 2013, mis en ligne

le 01 avril 2014, consulté le 22 août 2022. URL : http://journals.openedition.org/annuaire-cdf/1905

DOI : https://doi.org/10.4000/annuaire-cdf.1905 


\section{TABLEAU DES CHAIRES DEPUIS 1800}

\section{Chaire ancienne}

Chaire nouvelle

$1800^{1}$

Physique mathématique

Jacques-Antoine Cousin (1769-1800)

Histoire naturelle

Louis DAUBENTON (1778-1799)
Physique mathématique

Jean-Baptiste BIOT (1801-1862)

Histoire naturelle

Georges CUVIER (1800-1832)

\section{1}

Chimie

Nicolas VAUQUELIN (1801-1804)

\section{4}

Droit de la nature et des gens

Mathieu-Antoine BoUCHAUD (1773-1804)

Chimie

Nicolas VAUQUeLIN (1801-1804)

Création

Chimie

Jean DARCET (1774-1801)

Droit de la nature et des gens

Pierre DE PASTORET (1804-1821)

Chimie

Louis-Jacques THÉNARD (1804-1845)

Grec moderne

Jean-Baptiste D’ANSSE DE VILLOISON

(1804-1805)

\section{5}

Persan et Turc

Pierre RUFFIN (1784-1805)

Médecine

Jean-Nicolas CORVISART (1796-1804)
Persan

Antoine-Isaac SILVESTRE DE SACY (1806-1838)

Médecine

Jean-Noël HALLÉ (1805-1822)

1. L'année indiquée est celle de la délibération de l'Assemblée des Professeurs sur la création, le maintien ou la transformation de la chaire. 
Grec moderne

Jean-Baptiste D'ANSSE DE VILLOISON (1804-1805)

Turc

Pierre RUFFIN (1805-1822)

\section{7}

Astronomie

Jérôme DE LALANDE (1768-1807)

Astronomie

Jean-Baptiste DELAMBRE (1807-1822)

\section{9}

Éloquence latine

Charles-François DUPUIS (1787-1809)

Éloquence latine

Pierre GuÉROUlT (1809-1816)

\section{2}

Histoire et morale

Pierre-Charles LÉVESQUE (1791-1812)

Histoire et morale

Étienne CLAVIER (1812-1817)

\section{3}

Poésie latine

Jacques DELILLE (1778-1813)

Poésie latine

Pierre-François TISSOT (1813-1821)

\section{4}

Philosophie grecque et latine

Édouard BosQUILLON (1775-1814)

Littérature française

Antoine DE COURNAND (1784-1814)

Création

Création

Langue et philosophie grecques

Jean-François THUROT (1814-1832)

Littérature française

Stanislas ANDRIEUX (1814-1833)

Langues et littératures chinoise et tartare-mandchoue

Jean-Pierre ABEL-REMUSAT (1814-1832)

Langue et littérature sanscrites

Léonard DE CHÉZY (1815-1832)

\section{5}

\section{Mathématiques}

Antoine-Rémy MAUDUIT (1770-1815)

Mathématiques

Sylvestre-François LACROIX (1815-1843)

\section{6}

Éloquence latine

Pierre GUÉROULT (1809-1816)

Éloquence latine

Jean-Louis BURNOUF (1817-1844)

\section{7}

Histoire et morale

Étienne CLAVIER (1812-1817)

Histoire et morale

Pierre DAUNOU (1819-1830)

\section{9}

Hébreu

Prosper-Gabriel AUDRAN (1799-1819)

Hébreu

Étienne-Marc QUATREMÈRE (1819-1857) 


\section{1}

Droit de la nature et des gens

Pierre DE PASTORET (1804-1821)

Poésie latine

Pierre-François Tissot (révoqué) (1813-1821)

\section{Médecine}

Jean-Noël HALLÉ (1805-1822)

Turc

Pierre RUFFIN (1805-1822)

Astronomie

Jean-Baptiste DELAMBRE (1807-1822)

Physique générale et expérimentale Louis LEFÈVRE-GINEAU (révoqué) (1786-1823)

\section{Médecine}

René-Théophile LAENNEC (1822-1826)

Langue et littérature grecques Jean-Baptiste GAIL (1791-1829)

Histoire et morale

Pierre DAUNOU (1819-1830)

Poésie latine

Joseph NAUDET (1821-1830)

Médecine

Joseph RÉCAMIER (1827-1830)

Création

Création

Création
Droit de la nature et des gens

Xavier DE PORTETS (1822-1854)

Poésie latine

Joseph NAUDET (1821-1830)

\section{2}

\section{Médecine}

René-Théophile LAENNEC (1822-1826)

Turc

Daniel KIEFFER (1822-1833)

Astronomie

Jacques BINET (1823-1856)

\section{3}

Physique générale et expérimentale

André-Marie AMPÈRE (1824-1836)

\section{6}

Médecine

Joseph RÉCAMIER (1827-1830)

\section{9}

Langue et littérature grecques

Jean-François BOISSONADE (1829-1855)

\section{0}

Histoire et morale

Jean-Antoine LETRONNE (1831-1837)

Poésie latine

Pierre-François TISSOT (rétabli) (1830-1854)

\section{1}

Médecine

François MAGENDIE (1831-1855)

Économie politique Jean-Baptiste SAY (1831-1832)

Archéologie Jean-François CHAMPOLLION (1831-1832)

Histoire générale et philosophique des législations comparées

Eugène LERMINIER (1831-1849) 


\section{2}

Anatomie

Antoine PORTAL (1773-1832)

Histoire naturelle

Georges CUVIER (1800-1832)

Langues et littératures chinoise et tartare-mandchoue

Jean-Pierre ABEL-RÉMUSAT (1814-1832)

Langue et philosophie grecques

Jean-François THUROT (1814-1832)

Langue et littérature sanscrites

Léonard DE CHÉZY (1815-1832)

Économie politique

Jean-Baptiste SAY (1831-1832)

\section{Arabe}

Antoine CAussin DE Perceval (1784-1833)

Littérature française

Stanislas ANDRIEUX (1814-1833)

Turc

Daniel KIEFFER (1822-1833)

Physique générale et expérimentale André-Marie AMPÈRE (1824-1836)

\section{Archéologie}

Jean-François CHAMPOLLION (1831-1832)

Histoire et morale

Jean-Antoine LETRONNE (1831-1837)

Philosophie grecque et latine

Théodore JOUFFROY (1832-1837)

Création

Persan

Antoine-Isaac SILVESTRE DE SACY (1806-1838)

Chaire supprimée

Histoire naturelle, puis Histoire naturelle des corps inorganiques

Léonce ÉLIE DE BEAUMONT (1832-1874)

Langues et littératures chinoise et tartare-mandchoue

Stanislas JULIEN (1832-1873)

Philosophie grecque et latine

Théodore JOUFFROY (1832-1837)

Langue et littérature sanscrites

Eugène BURNOUF (1832-1852)

Économie politique

Pellegrino Rossi (1833-1840)

\section{3}

Arabe

Armand-Pierre CAUSSIN DE PERCEVAL (1833-1871)

Littérature française

Jean-Jacques AMPÈRE (1833-1853)

Turc

Alix DesGRANGES (1833-1854)

\section{6}

Physique générale et expérimentale Félix SAVART (1836-1841)

\section{7}

Archéologie

Jean-Antoine LETRONNE (1837-1848)

Histoire et morale

Jules MicheLET (1838-1852)

Philosophie grecque et latine Jules BARTHÉLÉMY SAINT-HILAIRE (1838-1852)

Histoire naturelle des corps organisés Georges DUVERNOY (1837-1855)

\section{8}

Persan

Amédée JAUBERT (1838-1847) 
1840

Économie politique

Pellegrino Rossi (1833-1840)

Création

Physique générale et expérimentale Félix SAVART (1836-1841)

Création

Création

Mathématiques

Sylvestre-François LACROIX (1843-1848)

Économie politique

Michel ChevaliER (1840-1879)

Langue et littérature slaves

Adam MiCKIEWICZ, chargé de cours (1840-1852)

\title{
1841
}

Physique générale et expérimentale

Henri-Victor RÉGNAULT (1841-1871)

Langues et littératures d'origine germanique

Philarète CHASLES (1841-1853)

Langues et littératures de l'Europe méridionale

Edgar QUINET (1841-1852)

\section{3}

Mathématiques

Guillaume LIBRI-CARUCCI (1815-1843)

\section{4}

Éloquence latine

Jean-Louis BURNOUF (1817-1844)

Création

Éloquence latine

Désiré NISARD (1844-1852)

Embryogénie comparée

Victor COSTE (1844-1873)

\section{5}

\section{Chimie}

Louis-Jacques THÉNARD (1804-1845)

Chimie minérale

Théophile-Jules Pelouze (1845-1850)

\section{7}

\author{
Persan \\ Jules MoHL (1850-1876)
}

\section{8}

Le 7 avril 1848, un décret du gouvernement provisoire supprima cinq chaires :

Économie politique, Droit de la nature et des gens, Législations comparées, Turc et Poésie latine,

pour en créer douze nouvelles destinées à instituer une École d'Administration dont l'existence fut éphémère :

Droit politique français et droit politique comparé, Jean REYNAUD

Droit international et histoire des traités, Alphonse DE LAMARTINE

Droit privé, Armand MARRAST

Droit criminel, Faustin HÉLIE

Économie générale et statistique de la population, Augustin SERRES

Économie générale et statistique de l'agriculture, Joseph DECAISNE 
Économie générale et statistique des mines, usines, arts et manufactures, Jean-Martial BINEAU

Économie générale et statistique des travaux publics, Alfred-Charles FRANQUET DE FRANQUEVILLE

Économie générale et statistique des finances et du commerce, Louis-Antoine GARNIERPAGÈS

Droit administratif, Louis-Marie DELAHAYE DE CORMENIN

Histoire des institutions administratives françaises et étrangères, Alexandre LEDRU-ROLLIN Mécanique, Jean-Victor PONCELET.

Le 14 novembre 1848, l'Assemblée Nationale rétablit les cinq chaires supprimées et leurs titulaires furent réintégrés au Collège.

\section{Chaire ancienne}

Histoire générale et philosophique des législations comparées

Édouard LABOULAYE (1849-1883)

Archéologie

Jean-Antoine LETRONNE (1837-1848)

\section{Mathématiques}

Guillaume LIBRI-CARUCCI (1843-1848)

Chimie minérale

Théophile-Jules PELOUZE (1845-1850)

Langue et littérature sanscrites

Eugène BURNOUF (1832-1852)

\section{Chaire nouvelle}

\section{9}

Histoire des législations comparées Eugène LERMINIER (1831-1849)

Archéologie

Charles LENORMANT (1849-1859)

\section{0}

Mathématiques

Joseph LIOUVILLE (1851-1882)

Chimie minérale

Antoine-Jérôme BALARD (1851-1876)

\section{2}

Langue et littérature sanscrites

Théodore PAVIE, chargé de cours (1853-1857)

Révocation de QUINET, MicheleT, MicKIEWICZ.

Langue et littérature slaves

Adam MicKIEWICZ, chargé de cours (1840-1852)

Éloquence latine

Désiré NISARD (1844-1852)
Langue et littérature slaves

Cyprien ROBERT, chargé de cours (1852-1857)

Éloquence latine

Wilhelm RINN (1853-1854)

\section{3}

\section{Littérature française}

Jean-Jacques AMPÈRE (1833-1853)

Philosophie grecque et latine Jules BARTHÉLÉMY SAINT-HILAIRE (1838-1852)
Langue et littérature françaises modernes Jean-Jacques AMPÈRE (1853-1864)

Philosophie grecque et latine

Émile SAISSET, chargé de cours (1853-1857) 
Fusion des chaires de:

- Langues et littératures de

l'Europe méridionale

Langues et littératures étrangères de l'Europe moderne

Edgar QUINET (révoqué) (1841-1852)

Philarète CHASLES (1853-1870)

- Langues et littératures d'origine germanique

Philarète CHASLES (1841-1853)

Création

Langue et littérature françaises du Moyen Âge

Paulin PARIS (1853-1872)

\section{4}

Droit de la nature et des gens

Xavier DE PORTETS (1822-1854)

Poésie latine

Pierre-François TISSOT (1830-1854)

Turc

Alix DesGranges (1833-1854)

Histoire et morale

Jules MichelET (révoqué) (1838-1852)

Éloquence latine

Wilhelm RINN (1853-1854)

Turc

Mathurin-Joseph COR (1854)

Droit de la nature et des gens

Adolphe FRANCK, chargé de cours (1854-1856), titulaire (1856-1887)

Poésie latine

Charles-Augustin DE SAINTE-BEUvE (1854-1869)

Turc

Mathurin-Joseph COR (1854)

Histoire et morale

Joseph GUIGNIAUT, chargé de cours

(1854-1857), titulaire (1857-1862)

Éloquence latine

Ernest HAVET (1854-1885)

Turc

Abel PAVET DE COURTEILle, chargé de cours (1854-1861), titulaire (1861-1889)

1855

Langue et littérature grecques

Jean-François BoISSONADE (1855-1892)

Médecine

François MAGENDIE (1831-1855)

Histoire naturelle des corps organisés

Georges DUVERNOY (1837-1855)

Langue et littérature grecques

Jean-Pierre Rossignol (1829-1855)

Médecine

Claude BERNARD (1855-1878)

Histoire naturelle des corps organisés

Pierre-Jean-Marie FLOURENS (1855-1867)

1857

Hébreu

Étienne-Marc QUATREMÈRE (1819-1857)

Langue et littérature slaves

Cyprien ROBERT, chargé de cours (1852-1857)

Philosophie grecque et latine

Émile SAISSET, chargé de cours (1853-1857)

Langues hébraïque, chaldaïque, et syriaque

Louis DuBEuX, chargé de cours (1857-1861)

Langue et littérature slaves

Alexandre CHODZKo, chargé de cours (1857-1883)

Philosophie grecque et latine

Charles LÉVÊQUE, chargé de cours (1857-1860), titulaire (1861-1900) 
Langue et littérature sanscrites

Théodore PAVIE, chargé de cours (1853-1857)
Langue et littérature sanscrites

Édouard FouCAUX, chargé de cours

(1857-1862), titulaire (1862-1894)

\section{0}

Astronomie

Jacques BINET (1823-1856)

Archéologie

Langues hébraïque, chaldaïque, et syriaque Louis DuBEUX, chargé de cours (1857-1861)

Création

Physique mathématique

Jean-Baptiste BIOT (1801-1862)

Histoire et morale

Joseph GUIGNIAUT (1857-1862)
Charles LENORMANT (1849-1859)

Mécanique céleste

Joseph SERRET (1861-1885)

Philologie et archéologie égyptiennes

Emmanuel DE RoUGÉ (1860-1872)

Langues hébraïque, chaldaïque, et syriaque Ernerst RENAN (1862-1864)

Épigraphie et antiquités romaines Léon RENIER (1861-1885)

\section{2}

Physique générale et mathématique Joseph BERTRAND (1862-1900)

Histoire et morale

Alfred MAURY, chargé de cours (1861), titulaire (1862-1892)

\section{4}

Langue et littérature françaises modernes Jean-Jacques AMPÈRE (1853-1864)

Langues hébraïque, chaldaïque, et syriaque Ernest RENAN (révoqué) (1862-1864)

Création

Langue et littérature françaises modernes Louis DE LOMÉNIE (1864-1878)

Langues hébraïque, chaldaïque, et syriaque Salomon MuNK (1864-1867)

Grammaire comparée

Michel BRÉAL, chargé de cours

(1864-1865), titulaire (1866-1905)

\section{5}

Création

Chimie organique

Marcelin BERTHELOT (1865-1907)

\section{9}

Poésie latine

Charles-Augustin DE SAINTE-BEuvE (1854-1869)

Histoire naturelle des corps organisés Pierre-Jean-Marie FLOURENS (1855-1867)

Poésie latine

Gaston BOISSIER (1869-1885)

Histoire naturelle des corps organisés Étienne-Jules MAREY (1869-1904)

\section{0}

Langues et littératures étrangères de l'Europe moderne

Philarète CHASLES (1853-1870)

Langues hébraïque, chaldaïque, et syriaque Salomon MuNK (1864-1867)

\section{Langues et littératures d'origine germanique \\ Philarète CHASLES (1870-1873)}

Langues hébräque, chaldaïque, et syriaque Ernest RENAN (rétabli) (1870-1892) 
Rétablissement

Arabe

Armand-Pierre CAUSSIN DE PERCEVAL (1833-1871)

Création

Physique générale et expérimentale Henri-Victor RÉGNAULT (1841-1871)

Langue et littérature françaises $d u$ Moyen Âge

Paulin PARIS (1853-1872)

Langues et littératures chinoise et tartare-mandchoue

Stanislas JULIEN (1832-1873)

Embryogénie comparée

Victor COSTE (1844-1873)

Philologie et archéologie égyptiennes

Emmanuel DE RoUGÉ (1860-1872)

Langues et littératures d'origine germanique

Philarète CHASLES (1870-1873)
Langues et littératures de l'Europe méridionale

Edgar QUINET (1870-1875)

\section{1}

Arabe

Charles-François DEFRÉMERY (1871-1883)

Histoire des doctrines économiques Émile LEVASSEUR (1871-1885)

\section{2}

Physique générale et expérimentale Élie MASCART (1872-1908)

Langue et littérature françaises $d u$ Moyen Âge

Gaston PARIS (1872-1903)

\section{3}

Langues et littératures chinoise et tartare-mandchoue

Léon D'HERVEY DE SAINT-DENYS (1874-1892)

Embryogénie comparée

Édouard BALBIANI (1874-1899)

Philologie et archéologie égyptiennes

Gaston MASPERO (1874-1916)

Langues et littératures d'origine germanique

Guillaume GUIZOT (1874-1892)

\section{4}

Histoire naturelle des corps inorganiques Léonce ÉLIE DE BEAUMONT (1832-1874)

Création

Création

Histoire naturelle des corps inorganiques Charles SAINTE-Claire DeviLle (1875-1876)

Histoire de la philosophie moderne Jean NOURRISSON (1874-1899)

Philologie et archéologie assyriennes Jules OPPERT (1874-1905)

\section{5}

Langues et littératures de l'Europe méridionale

Edgar QUINET (1870-1875)

Création
Langues et littératures de l'Europe méridionale

Paul MEYER (1876-1906)

Anatomie générale

Louis RANVIER (1875-1911) 
1876

Persan

Jules MoHL (1850-1876)

Chimie minérale

Antoine-Jérôme BALARD (1851-1876)

Histoire naturelle des corps inorganiques

Charles SAINTE-ClaIREDEVILLE (1875-1876)
Persan

Adrien BARBIER DEMEYNARD (1876-1885)

Chimie minérale

Paul SCHÜTZENBERGER (1876-1897)

Histoire naturelle des corps inorganiques

Ferdinand FOUQUÉ (1877-1904)

\section{7}

Création

Médecine

Claude BERNARD (1855-1878)

Langue et littérature françaises modernes Louis DE LOMÉNIE (1864-1878)

Création

Économie politique

Michel CHEVALIER (1840-1879)

Langue et littérature françaises modernes Paul ALBERT (1878-1880)

Création

Mathématiques

Joseph LIOUVILLE (1851-1882)

Esthétique et histoire de l'art

Charles BLANC (1878-1882)

Création

Histoire des législations comparées

Édouard LABOULAYE (1849-1883)

Arabe

Charles-François DEFRÉMERY (1871-1883)
Épigraphie et antiquités grecques

Paul FOUCART (1877-1926)

\section{8}

\author{
Médecine \\ Charles-Édouard BROWN-SÉQUARD \\ (1878-1894)
}

Langue et littérature françaises modernes Paul ALBERT (1878-1880)

Esthétique et histoire de l'art

Charles BLANC (1878-1882)

\section{0}

Économie politique

Paul LEROY-BEAULIEU (1880-1916)

Langue et littérature françaises modernes Émile DESCHANEL (1881-1903)

Histoire des religions

Albert RÉVILLE (1880-1906)

\section{2}

Mathématiques

Camille JORDAN (1883-1912)

Esthétique et histoire de l'art

Eugène GuILLAUME (1882-1905)

Langues et littératures celtiques

Henry D'ARBoIS DE JUBAINVILLE (1882-1910)

\section{3}

Histoire des législations comparées Jacques FLACH (1884-1919)

Arabe

Stanislas GUYARD (1884) 


\section{4}

Langue et littérature slaves

Alexandre CHODZKo, chargé de cours (1857-1883)

Arabe

Stanislas GUYARD (1884)

Éloquence latine

Ernest HAVET (1854-1885)

Mécanique céleste

Joseph SERRET (1861-1885)

Épigraphie et antiquités romaines

Léon RENIER (1861-1885)

Poésie latine

Gaston BOISSIER (1869-1885)

Histoire des doctrines économiques

Émile LEVASSEUR (1871-1885)

Persan

Adrien BARBIER DE MEYNARD (1876-1885)
Langues et littératures slaves

Louis LÉGER (1885-1923)

Arabe

Adrien BARBIER DE MEYNARD (1885-1908)

\section{5}

Philologie latine

Louis HAVET (1885-1925)

Mécanique analytique et mécanique céleste Maurice LÉVY (1885-1908)

Épigraphie et antiquités romaines

Ernest DESJARDINS (1886)

Histoire de la littérature latine

Gaston BOISSIER (1885-1906)

Géographie, histoire, et statistiques économiques

Émile LEVASSEUR (1885-1911)

Langues et littératures de la Perse James DARMESTETER (1885-1894)

\section{6}

Épigraphie et antiquités romaines

Ernest DESJARDINS (1886)

Épigraphie et antiquités romaines

René CAGNAT (1887-1930)

\section{7}

Droit de la nature et des gens

Adolphe FRANCK (1856-1887)

Psychologie expérimentale et comparée

Théodule RIBOT (1888-1901)

\section{0}

Turc

Abel Pavet De CourTeille (1861-1889)

Épigraphie et antiquités sémitiques

Charles CleRMONT-GANNEAU (1890-1923)

\section{2}

Histoire et morale

Alfred MAURY (1862-1892)

Création

Géographie historique de la France Auguste LoNGNON (1892-1911)

Histoire générale des sciences Pierre LAFFITTE (1892-1903)

\section{3}

Langue et littérature grecques Jean-Pierre RossigNOL (1855-1892)

Langues hébraïque, chaldaïque et syriaque

Ernest RENAN (1870-1892)
Langue et littérature grecques

Maurice CROISET (1893-1930)

Langues et littératures hébraïque, chaldä̈que, et syriaque

Philippe BERGER (1893-1910) 
Langues et littératures d'origine germanique Guillaume GuIZOT (1874-1892)

Langues et littératures chinoise et tartare-mandchoue

Léon D'HERVEY DE SAINT-DENYS (1874-1892)

Langue et littérature sanscrites

Édouard FoUCAUX (1862-1894)

Médecine

Charles-Édouard BROWN-SÉQUARD (1878-1894)

Langues et littératures de la Perse James DARMESTETER (1885-1894)

Chimie minérale

Paul SCHÜTZENBERGER (1876-1897)

Création

Histoire de la philosophie moderne Jean NOURRISSON (1874-1899)

Embryogénie comparée

Édouard BALBIANI (1874-1899)

Philosophie grecque et latine Charles LÉVÊQUE (1861-1900)

Physique générale et mathématique Joseph BERTRAND (1862-1900)

Psychologie expérimentale et comparée Théodule RIBOT (1888-1901)

Création

Langue et littérature françaises du Moyen Âge

Gaston PARIS (1872-1903)
Langues et littératures d'origine germanique Arthur CHUQUET (1893-1925)

Langues et littératures chinoise et tartare-mandchoue

Édouard CHAVANNES (1893-1918)

\section{4}

Langue et littérature sanscrites Sylvain LÉVI (1894-1935)

Médecine

Arsène D'ARSONVAL (1894-1930)

Langue et littérature araméennes Rubens DuVAL (1894-1907)

\section{7}

Chimie minérale

Henri LE CHATELIER (1898-1907)

Philosophie sociale

Jean IZOULET (1897-1929)

\section{9}

Philosophie moderne

Gabriel TARDE (1900-1904)

Embryogénie comparée

Félix HENNEGUY (1900-1928)

\section{0}

Philosophie grecque et latine Henri BERGSON (1900-1904)

Physique générale et mathématique Marcel BRILLOUIN (1900-1931)

\section{1}

Psychologie expérimentale et comparée Pierre JANET (1902-1934)

\section{2}

Sociologie et sociographie musulmanes Alfred LE CHATELIER (1902-1925)

\section{3}

Langue et littérature françaises du Moyen Âge

Joseph BÉDIER (1903-1936) 
Histoire générale des sciences

Pierre LAFFITTE (1892-1903)

Création

Étienne-Jules MAREY (1869-1904)

Histoire naturelle des corps inorganiques Ferdinand FOUQUÉ (1877-1904)

Langue et littérature françaises modernes Émile DESCHANEL (1881-1903)

Philosophie moderne

Gabriel TARDE (1900-1904)

Grammaire comparée

Michel BRÉAL (1866-1905)

Philologie et archéologie assyriennes Jules OPPERT (1874-1905)

Esthétique et histoire de l'art Eugène GuILLAUME (1882-1905)

Philosophie grecque et latine

Henri BERGSON (nommé en 1904 titulaire de la chaire dePhilosophie moderne) (1900-1904)

Langues et littératures de l'Europe méridionale

Paul MEYER (1876-1906)

Histoire des religions

Albert RÉVILLE (1880-1906)

Histoire de la littérature latine

Gaston BOISSIER (1885-1906)

Chimie organique

Marcelin BERTHELOT (1865-1907)

Chimie minérale

Henri LE CHATELIER (1898-1907)

Pathologie générale et comparée Albert CHARRIN (1903-1907)

Création
Histoire générale des sciences

Grégoire WyROUBOFF (1903-1913)

Pathologie générale et comparée

Albert CHARRIN (1903-1907)

\section{4}

Histoire naturelle des corps organisés Nicolas FRANÇOIS-FRANCK (1905-1921)

Histoire naturelle des corps inorganiques Auguste MicHEL-LÉVY (1905-1911)

Langue et littérature françaises modernes Abel LEFRANC (1904-1937)

Philosophie moderne

Henri BERGSON (1904-1921)

\section{5}

Grammaire comparée

Antoine MEILLET (1906-1936)

Philologie et archéologie assyriennes

Charles Fossey (1906-1939)

Esthétique et histoire de l'art Georges LAFENESTRE (1905-1919)

Histoire et antiquités nationales

Camille JULLIAN (1905-1930)

1906

Langues et littératures de l'Europe méridionale

Alfred MOREL-FATIO (1907-1924)

Histoire des religions

Jean RÉVILLE (1907-1908)

Histoire de la littérature latine

Paul MonCEAUX (1907-1934)

1907

Chimie organique

Émile JUNGFLEISCH (1908-1916)

Chimie minérale

Camille MAtignon (1908-1934)

Biologie générale

Émile GLEY (1908-1930)

Histoire du travail

Georges RENARD (1907-1930) 


\section{8}

Physique générale et expérimentale

Élie MASCART (1872-1908)

Arabe

Adrien BARBIER DEMEYNARD (1885-1908)

Langue et littérature araméennes

Rubens DuVAL (1895-1907)

Histoire des religions

Jean RÉVILLE (1907-1908)
Physique générale et expérimentale

Paul LANGEVIN (1909-1946) (révoqué en 1940, réintégré en 1944)

Langue et littérature arabes

Paul CASANOVA (1909-1926)

Numismatique de l'Antiquité et du Moyen Âge

Ernest BABELON (1908-1924)

Histoire des religions

Alfred LOISY (1909-1932)

\section{9}

Mécanique analytique et mécanique céleste Maurice LÉVY (1885-1908)

\section{Langues et littératures celtiques}

Henry D'ARBoIS DE JUBAINVILLE (1882-1910)

Langues et littératures hébräque, chaldaïque, et syriaque

Philippe BERGER (1893-1910)

Anatomie générale

Louis RANVIER (1875-1911)

Mathématiques

Camille JORDAN (1883-1912)

Géographie, histoire et statistiques économiques

Émile LEVASSEUR (1885-1911)

Géographie historique de la France Auguste LongNON (1892-1911)

Histoire naturelle des corps inorganiques Lucien CAYEUX (1912-1936)

Création temporaire (fondation Albert Kahn)

Histoire générale des sciences

Grégoire WyROUBOFF (1903-1913)
Mécanique analytique et mécanique céleste Jacques HADAMARD (1909-1937)

\section{0}

Langues et littératures celtiques

Joseph LOTH (1910-1930)

\section{1}

Langues, histoire et archéologie de l'Asie centrale

Paul PELLIOT (1911-1945)

\section{2}

Histologie comparée

Jean NAGEOTTE (1912-1937)

Mathématiques

Georges HUMBERT (1912-1921)

Étude des faits économiques et sociaux

Marcel MARION (1912-1932)

Histoire de l'Afrique du Nord

Stéphane GSELL (1912-1932)

Géologie

Auguste MiCHEL-LÉVY (1905-1911)

Géographie humaine

Jean BRUNHES (1912-1930)

\section{4}

Chaire supprimée 


\section{6}

Philologie et archéologie égyptiennes Gaston MASPERO (1874-1916)

Chimie organique

Émile JUNGFLEISCH (1908-1916)

Création

(fondation de la Ville de Paris)

Économie politique

Paul LEROY-BEAULIEU (1880-1916)

Langues et littératures chinoise et tartare-mandchoue

Édouard CHAVANNES (1893-1918)

Esthétique et histoire de l'art

Georges LAFENESTRE (1905-1919)

Histoire des législations comparées Jacques FLACH (1884-1919)

Création

Création temporaire

Philosophie moderne

Henri BERGSON (1904-1921)

Histoire naturelle des corps organisés

Nicolas FRANÇOIS-FRANCK (1905-1921)

Mathématiques

Georges HUMBERT (1912-1921)

Création

Histoire des sciences

Pierre BouTROUX (1920-1922)

Langues et littératures slaves

Louis LÉGER (1885-1923)

Épigraphie et antiquités sémitiques

Charles Clermont-GANNEAU (1890-1923)
Chaire supprimée

Chaire supprimée

Prévoyance et assistance sociales

Édouard FUSTER (1917-1935)

\section{7}

Chimie organique

Charles MOUREU (1917-1929)

1919

Langue et littérature chinoises

Henri MASPERO (1921-1945)

Histoire de l'art français

André MICHEL (1920-1925)

1920

Histoire des sciences

Pierre BoUTROUX (1920-1922)

Histoire et philologie indochinoises

Louis FINOT (1920-1930)

Enseignement de la coopération

Charles GIDE (1921-1930)

1921

Philosophie

Édouard LEROY (1921-1940)

Histoire naturelle des corps organisés

André MAYER (1922-1946)

Mathématiques

Henri LEBESGUE (1921-1941)

Histoire coloniale

Alfred MARTINEAU (1921-1935)

1922

Égyptologie

Alexandre MORET (1923-1938)

1923

Langues et littératures slaves

André MAZON (1923-1951)

Physiologie des sensations

Henri PIÉRON (1923-1951) 
Création

Création temporaire

Langues et littératures de l'Europe méridionale

Edmond FARAL (1925-1954)

Numismatique de l'Antiquité et du Moyen Âge

Théodore REINACH (1924-1928)

Philologie latine

Louis HAVET (1885-1925)

Langues et littératures d'origine germanique

Arthur CHUQUET (1893-1925)

Phonétique expérimentale

Jean RousSELOT (1923-1924)

Création

Épigraphie et antiquités grecques Paul FOUCART (1877-1926)

Sociologie et sociographie musulmanes Alfred LE CHATELIER (1902-1925)

Langue et littérature arabes

Paul CASANOVA (1909-1926)

Histoire de l'art français

André MICHEL (1920-1925)

Embryogénie comparée

Félix HENNEGUY (1900-1928)

Philosophie sociale

Jean IZOULET (1897-1929)

Chimie organique

Charles MOUREU (1917-1929)
Phonétique expérimentale

Jean RouSSELOT (1923-1924)

Études coloniales, protistologie pathologique

Louis NATTAN-LARRIER (1923-1943)

\section{4}

Littérature latine du Moyen Âge Alfred MOREL-FATIO (1907-1924)

Numismatique de l'Antiquité

Ernest BABELON (1908-1924)

\section{5}

Histoire des littératures comparées de l'Europe méridionale et de l'Amérique latine

Paul HAZARD (1925-1944)

Langues et littératures d'origine germanique

Charles ANDLER (1926-1933)

Histophysiologie

Justin JOLLY (1925-1940)

Épidémiologie

Hyacinthe VINCENT (1925-1936)

\section{6}

Épigraphie grecque

Maurice HoLlEAUX (1927-1932)

Sociologie et sociographie musulmanes Louis MASSIGNON (1926-1954)

Langue et littérature arabes William MARÇAIS (1927-1943)

Esthétique et histoire de l'art

Gabriel MiLlET (1926-1937)

\section{8}

Embryogénie comparée

Emmanuel FAURÉ-FrÉMIET (1928-1954)

\section{9}

Sociologie

Marcel MAUSS (1931-1942)

Chimie organique

Marcel DELÉPINE (1930-1941) 
Numismatique de l'Antiquité

Théodore REINACH (1924-1928)

Création

Histoire et philologie indochinoises Louis FINOT (1920-1930)

Épigraphie et antiquités romaines René CAGNAT (1887-1930)

Langue et littérature grecques Maurice CROISET (1893-1930)

Médecine

Arsène D'ARSONVAL (1894-1930)

Physique générale et mathématique Marcel BRILLOUIN (1900-1931)

Histoire du travail

Georges RENARD (1907-1930)

Biologie générale

Émile GLEY (1908-1930)

Langues et littératures celtiques Joseph LOTH (1910-1930)

Création

Histoire des religions

Alfred LOISY (1909-1932)

Histoire de l'Afrique du Nord

Stéphane GSELL (1912-1932)

Étude des faits économiques et sociaux Marcel MARION (1912-1932)

Création temporaire (fondation de la Ville de Paris)

Langues et littératures d'origine germanique

Charles ANDLER (1926-1933)

Épigraphie grecque

Maurice HolleauX (1927-1932)

Création (pour Albert EINSTEIN, qui ne devait jamais l'occuper)
Préhistoire

Henri BREUIL (1929-1947)

Mécanique animale appliquée de l'aviation Antoine MAGNAN (1929-1938)

\section{0}

Histoire et philologie indochinoises Jean PRZYLUSKI (1931-1944)

\section{1}

Civilisation romaine

Eugène ALBERTINI (1932-1941)

Langue et littérature grecques

Émile BouRGUET (1932-1938)

Médecine

Charles NiCOLLE (1932-1936)

Physique théorique

Léon BRILLOUIN (1932-1949)

Histoire du travail

François SIMIAND (1932-1935)

Biologie générale

Jacques DUCLAUX (1931-1948)

Histoire de la philosophie au Moyen Âge Étienne GILSON (1932-1950)

Civilisation américaine

Bernard FAŸ (1932-1945)

\section{2}

Histoire de la civilisation moderne Lucien FEBVRE (1933-1949)

Histoire ancienne de l'Orient sémitique Isidore LÉVY (1932-1941)

Géographie économique et politique André SIEGFRIED (1933-1946)

Histoire de l'art monumental Paul LÉON (1932-1944)

\section{3}

Langues et littératures d'origine germanique

Ernest TONNELAT (1934-1948)

Histoire des religions

Jean BARUZI (1933-1951)

Physique mathématique 
Suppression par décret des chaires de :

Psychologie expérimentale et comparée

Pierre JANET (1902-1934)

Histoire de la littérature latine

Paul MonCEAUX (1907-1934)

Chimie minérale

Camille MATIGNON (1908-1934)

1935

Histoire et antiquités nationales

Camille JULLIAN (1905-1930)

Prévoyance et assistance sociales

Édouard FUSTER (1917-1935)

Histoire du travail

François SIMIAND (1932-1935)

Antiquités nationales

Albert GRENIER (1936-1948)

Psychologie et éducation de l'enfance

Henri WALLON (1937-1949)

Histoire du travail

Émile COORNAERT (1936-1957)

\section{6}

Langue et littérature sanscrites

Sylvain LÉvI (1894-1935)

Médecine

Charles NICOLLE (1932-1936)

Chimie nucléaire

Frédéric JoLIOT (1937-1958)

Langue et littérature sanscrites Jules BLOCH (1937-1951)

\section{7}

Langue et littérature françaises du Moyen Âge Histoire du vocabulaire français Joseph BÉDIER (1903-1936)

Mario RoQuEs (1937-1946)

Langue et littérature françaises modernes

Poétique

Paul VALÉRY (1937-1945)

Abel LEFRANC (1904-1937)

Grammaire comparée

Antoine MeILlET (1906-1936)

Grammaire comparée

Émile BENVENISTE (1937-1972)

Géologie

Lucien CAYEUX (1912-1936)

Histoire coloniale

Géologie méditerranéenne

Paul FALLOT (1938-1960)

Alfred MARTINEAU (1921-1935)

Épidémiologie

Hyacinthe VINCENT (1925-1936)

Histoire de la colonisation

Edmond CHASSIGNEUX (1939-1946)

Médecine

René LERICHE (1937-1950)

\section{8}

Mécanique analytique et mécanique céleste Jacques HADAMARD (1909-1937)

Histologie comparée

Jean NAGEOTTE (1912-1937)

Égyptologie

Alexandre MORET (1923-1938)
Mathématique et mécanique Szolem MANDELBROJT (1938-1972)

Morphologie expérimentale et endocrinologie

Robert COURRIER (1938-1966)

Égyptologie

Pierre LACAU (1938-1947) 
Esthétique et histoire de l'art

Gabriel MiLLET (1926-1937)

Mécanique animale appliquée à l'aviation Antoine MAGNAN (1929-1938)

Langue et littérature grecques

Émile BOURGUET (1932-1938)

Philologie et archéologie assyriennes

Charles FossEY (1906-1939)

Physique générale et expérimentale

Paul LANGEVIN (1909-1946) (révoqué en 1940, réintégré en 1944)

Philosophie

Édouard LEROY (1921-1940)

Mathématiques

Henri LEBESGUE (1921-1941)

Histophysiologie

Justin JOLLY (1925-1940)

Chimie organique

Marcel DELÉPINE (1930-1941)

Civilisation romaine

Eugène ALBERTINI (1932-1941)

Histoire des littératures comparées de l'Europe méridionale et de l'Amérique latine

Paul HAZARD (1925-1944)

Sociologie

Marcel MAUSS (1931-1942)

Esthétique et histoire de l'art

Henri FoCILLON (révoqué) (1938-1942)

Mathématique et mécanique

Szolem MANDELBROJT (1938-1972) (révoqué en 1942, réintégré en 1944)

Langue et littérature chinoises

Henri MASPERO (1921-1945)

Langue et littérature arabes

William MARÇAIS (1927-1943)

\section{$1945^{2}$}

Esthétique et histoire de l'art

Henri FoCILLON (1938-1942)

Aérolocomotion mécanique et biologique Étienne CEHMichen (1939-1955)

Épigraphie et antiquités grecques

Louis ROBERT (1939-1974)

\section{1}

Histoire des arts de l'Orient musulman

Albert GABRIEL (1941-1953)

Physique générale et expérimentale

Maurice Debroglie (1942-1944), puis à nouveau Paul LANGEVIN

Philosophie

Louis LAVELLE (1941-1951)

Affectation réservée

Radiobiologie expérimentale

Antoine LACASSAGNE (1941-1951)

Chimie organique

Charles DUFRAISSE (1942-1955)

Civilisation romaine

André PIgANIOL (1942-1954)

\section{3-1944}

Langues et littératures de la péninsule ibérique et de l'Amérique latine

Marcel BATAILlON (1945-1965)

Psychologie collective

Maurice HALBWACHS (1944-1945)

Histoire de la langue latine

Alfred ERNOUT (1944-1951)

Physique cosmique

Alexandre DAUVILLIER (1944-1962)
Langue et littérature chinoises
Paul DemiÉville (1946-1964)
Histoire du monde arabe
Jean SAUVAGET (1946-1950)

2. L'année indiquée est celle de la délibération de l'Assemblée des Professeurs; l'arrêté ministériel déclarant la vacance de la chaire, postérieur de quelques mois, peut être parfois daté de l'année suivante. 
Histoire ancienne de l'Orient sémitique Isidore LÉVY (1932-1941)

Physique mathématique

(chaire créée pour A. Einstein en 1933)

Poétique

Paul VALÉRY (1937-1945)

Psychologie collective

Maurice HALBWACHS (1944-1945)

Physique générale et expérimentale

Paul LANGEVIN (1909-1946)

Langues, histoire et archéologie de l'Asie centrale

Paul PELLIOT (1911-1945)

Histoire naturelle des corps organisés André MAYER (1922-1946)

Histoire et philologie indochinoises Jean PRZYLUSKI (1931-1944)

Civilisation américaine

Bernard FAŸ (révoqué) (1932-1945)

Géographie économique et politique André SIEGFRIED (1933-1946)

Histoire du vocabulaire français Mario RoQUES (1937-1946)

Création

Préhistoire

Henri BREUIL (1929-1947)

Égyptologie

Pierre LACAU (1938-1947)

Histoire de la colonisation

Edmond CHASSIGNEUX (1939-1946)

Biologie générale

Jacques DUCLAUX (1931-1948)

Langues et littératures d'origine germanique

Ernest TONNELAT (1934-1948)
Philologie et archéologie assyrobabyloniennes

Édouard DHORME (1945-1951)

Physique atomique et moléculaire Francis PERRIN (1946-1972)

Histoire des créations littéraires en France Jean POMMIER (1946-1964)

Histoire de la civilisation italienne Augustin RENAUDET (1946-1950)

\section{6}

Théorie des équations différentielles et fonctionnelles

Jean LERAY (1947-1978)

Archéologie paléochrétienne et byzantine André GRABAR (1946-1966)

Biochimie générale et comparée Jean RocHE (1947-1972)

Histoire et philologie indochinoises Émile GASPARDONE (1946-1965)

Histoire de la civilisation de l'Amérique du Nord Marcel GIRAUD (1947-1971)

Étude du monde tropical Pierre GouROU (1947-1970)

Langue et littérature françaises du Moyen Âge

Félix LECOY (1947-1974)

Civilisations de l'Extrême-Orient Paul Mus (1946-1969)

\section{7}

Géographie historique de la France Roger DION (1948-1968)

Égyptologie

Pierre MONTET (1948-1956)

Histoire de l'expansion de l'Occident Robert MonTAGNÉ (1948-1954)

\section{8}

Neurophysiologie générale Alfred FESSARD (1949-1971)

Langues et littératures d'origine germanique

Fernand Mossé (1949-1956) 
Antiquités nationales

Albert GRENIER (1936-1948)

Physique théorique

Léon BRILLOUIN (1932-1949)

Histoire de la civilisation moderne Lucien FEBVRE (1933-1949)

Psychologie et éducation de l'enfance Henri WALLON (1937-1949)

\section{Médecine}

René LERICHE (1937-1950)

Histoire de la civilisation italienne Augustin RENAUDET (1946-1950)

Histoire du monde arabe Jean SAuVAGET (1946-1950)

Création

(fondation de la ville de Paris)

Langues et littératures slaves

André MAZON (1923-1951)

Physiologie des sensations

Henri PIÉRON (1923-1951)

Histoire de la philosophie au Moyen Âge Étienne GILSON (1932-1950)

Histoire des religions

Jean BARUZI (1933-1951)

Langue et littérature sanscrites

Jules BLOCH (1937-1951)

Philosophie

Louis LAVELLE (1941-1951)

Histoire de la langue latine

Alfred ERNOUT (1944-1951)

Philologie et archéologie assyro-babyloniennes

Édouard DHORME (1945-1951)

Radiobiologie expérimentale

Antoine LACASSAGNE (nommé en 1951, titulaire de la chaire de

Médecine expérimentale créée l'année précédente)
Civilisation indo-européenne

Georges DuMÉzIL (1949-1968)

1949

Physique théorique

Jean LAVAL (1950-1970)

Histoire de la civilisation moderne Fernand BRAUDEL (1950-1972)

Psychologie des arts plastiques René HuYGHE (1951-1976)

\section{0}

Médecine expérimentale Antoine LACASSAGNE (1951-1954)

Littérature et civilisation italiennes André PÉZARD (1951-1963)

Langue et littérature arabes Gaston WIET (1951-1959)

Histoire et structure sociales de Paris et de la région parisienne Louis CHEVALIER (1952-1981)

\section{1}

Langues et littératures slaves André VAILLANT (1952-1962)

Physique mathématique André LICHNEROWICZ (1952-1986)

Histoire et technologie des systèmes philosophiques

Martial GUÉROULT (1951-1962)

Histoire des religions

Henri-Charles PUECH (1952-1972)

Langues et littératures de l'Inde Jean FILLIOZAT (1952-1978)

Philosophie

Maurice MERLEAU-PONTY (1952-1961)

Littérature latine

Pierre COURCELLE (1952-1980)

Assyriologie

René LABAT (1952-1974)

Histophysiologie

Jacques BENOIT (1952-1966) 


\section{3}

Archéologie de l'Asie occidentale Albert GABRIEL (1941-1953)

Littérature latine du Moyen Âge Edmond FARAL (1925-1954)

Embryogénie comparée

Emmanuel FAURÉ-FrÉMIET (1928-1954)

Civilisation romaine

André PIGANIOL (1942-1954)

Médecine expérimentale

Antoine LACASSAGNE (1951-1954)

Sociologie et sociographie musulmanes Louis MASSIGNON (1926-1954)

Aérolocomotion mécanique et biologique Étienne EHMICHEN (1939-1955)

Chimie organique

Charles DUFRAISSE (1942-1955)

Histoire de l'expansion de l'Occident Robert MONTAGNÉ (1948-1954)

Égyptologie

Pierre MONTET (1948-1956)

Langues et littératures d'origine germanique

Fernand MossÉ (1949-1956)

Histoire du travail

Émile COORNAERT (1936-1957)

Chimie nucléaire

Frédéric JOLIOT (1937-1958)

Création

Langue et littérature arabes

Gaston WIET (1951-1959)
Histoire des arts de l'Orient musulman

Claude SCHAEFFER-FORRER (1954-1969)

\section{4}

Analyse des faits économiques et sociaux François PERROUX (1955-1974)

Embryologie expérimentale Étienne WOLFF (1955-1974)

Civilisation romaine

Jean GAGÉ (1955-1972)

Médecine expérimentale

Charles OBERLING (1955-1960)

\section{5}

Sociologie musulmane

Henri LAOUST (1956-1975)

Algèbre et géométrie

Jean-Pierre SERRE (1956-1994)

Chimie organique des hormones

Alain HOREAU (1956-1980)

Histoire sociale de l'Islam contemporain Jacques BERQUE (1956-1981)

\section{6}

Philologie et archéologie égyptiennes Étienne DRIOTON (1957-1960)

\section{7}

Langues et littératures d'origine germanique

Robert MINDER (1957-1973)

\section{8}

Démographie sociale : la vie des populations

Alfred SAUVY (1959-1969)

Physique nucléaire

Louis LEPRINCE-RINGUET (1959-1972)

Anthropologie sociale

Claude LÉVI-STRAUSS (1959-1982)

\section{9}

Magnétisme nucléaire

Anatole ABRAGAM (1960-1985) 
1960

Géologie méditerranéenne

Paul FALLOT (1938-1960)

Médecine expérimentale

Charles OBERLING (1955-1960)

Philologie et archéologie égyptiennes

Étienne DRIOTON (1957-1960)

Philosophie

Maurice MERLEaU-PONTY (1952-1961)

Histoire et technologie des systèmes philosophiques

Martial GUÉROULT (1951-1962)

Langues et littératures slaves

André VaiLlant (1952-1962)

Physique cosmique

Alexandre DAUVILLIER (1944-1962)

Langue et littérature chinoises

Paul DÉMIÉvILLE (1946-1964)

Histoire des créations littéraires en France Georges BLIN (1965-1988)

Littérature et civilisation italiennes André PÉZARD (1951-1963)

Création

Création

Langues et littératures de la péninsule ibérique et de l'Amérique latine

Marcel BATAILLON (1945-1965)

Histoire et philologie indochinoises

Émile GASPARDONE (1946-1965)
Méthodes physiques de l'astronomie

André LALLEMAND (1961-1974)

Médecine expérimentale

Bernard HALPERN (1961-1975)

Philologie et archéologie égyptiennes

Georges POSENER (1961-1978)

\section{1}

Philosophie de la connaissance

Jules VuILLEMIN (1962-1990)

\section{2}

Histoire de la pensée philosophique Jean HyPPOLITE (1963-1968)

Hébreu et araméen

André DUPONT-SOMMER (1963-1971)

\section{3}

Astrophysique théorique

Jean-Claude PECKER (1964-1988)

Histoire et civilisations de l'Asie centrale

Louis HAMBIS (1965-1977)

Littérature française moderne

Jean POMMIER (1946-1964)

Archéologie et histoire de la Gaule

Paul-Marie Duval (1964-1982)

Physique théorique des particules élémentaires

Jacques PRENTKI (1965-1983)

Génétique cellulaire

François JАСОВ (1965-1991)

\section{5}

Langues et littératures de la péninsule ibérique et de l'Amérique latine

Israël RÉVAH (1966-1973)

Étude du monde chinois : institutions et concepts

Rolf A. STEIN (1966-1981) 


\section{7}

Morphologie expérimentale et endocrinologie Robert COURRIER (1938-1966)

Physiologie cellulaire

Archéologie paléochrétienne et byzantine François MOREL (1967-1993)

André GRABAR (1946-1966)

Histophysiologie

Jacques BENOIT (1952-1966)

Histoire et civilisation de Byzance

Paul LEMERLE (1967-1973)

Biologie moléculaire

Jacques MONOD (1967-1973)

\section{8}

Civilisation indo-européenne

Georges DuMÉZIL (1949-1968)

Préhistoire

André LEROI-GOURHAN (1969-1982)

\section{9}

Géographie historique de la France

Roger DION (1948-1968)

Géographie du continent européen

Maurice LELANNOU (1969-1976)

Archéologie de l'Asie occidentale

Claude SCHAEFFER-FORRER (1954-1969)

Histoire des sociétés médiévales

Georges DUBY (1970-1991)

Démographie sociale : la vie des populations Alfred SAUVY (1959-1969)

Sociologie de la civilisation moderne Raymond ARON (1970-1978)

Histoire de la pensée philosophique Jean HyPPOLITE (1963-1968)

Histoire des systèmes de pensée Michel FouCAULT (1970-1984)

\section{0}

Civilisations de l'Extrême-Orient

Paul Mus (1946-1969)

Étude du monde tropical

Pierre GouROU (1947-1970)

Physique théorique

Jean LAVAL (1950-1970)

Art et civilisation de la Renaissance en Italie

André CHASTEL (1970-1984)

Étude du Bouddhisme

André BAREAU (1971-1991)

Physique de la matière condensée

Pierre-Gilles DE GENNES (1971-2004)

\section{1}

Histoire de la civilisation de l'Amérique du Nord Marcel GIRAUD (1947-1971)

Neurophysiologie générale

Alfred FESSARD (1949-1971)

Hébreu et araméen

André DUPONT-SOMMER (1963-1971)

Anthropologie physique

Jacques RUFFIÉ (1972-1992)

Neurophysiologie

Yves LAPORTE (1972-1991)

Hébreu et araméen

André CAQUOT (1972-1994)

\section{2}

Physique atomique et moléculaire Francis PERRIN (1946-1972)

Biochimie générale et comparée Jean RoCHE (1947-1972)

Physique atomique et moléculaire Claude COHEN-TANNOUDJI (1973-2004)

Biochimie cellulaire François GROS (1973-1996) 
Physique nucléaire

Louis LEPRINCE-RINGUET (1959-1972)
Physique corpusculaire

Marcel FROISSART (1973-2004)

\section{3}

Grammaire comparée

Émile BENVENISTE (1937-1972)

Mathématique et mécanique

Szolem MANDELBROJT (1938-1972)

Histoire de la civilisation moderne Fernand BRAUDEL (1950-1972)

Histoire des religions

Civilisation romaine

Jean GAGÉ (1955-1972)

Langues et littératures d'origine germanique Robert MiNDER (1957-1973)

Langues et littératures de la péninsule ibérique et de l'Amérique latine

Israël RÉVAH (1966-1973)

Biologie moléculaire

Jacques MONOD (1967-1973)

Histoire et civilisation de Byzance

Paul LEMERLE (1967-1973)
Henri-Charles PUECH (1952-1972)

Langues et civilisation de l'Asie Mineure Emmanuel LAROCHE (1973-1985)

Analyse mathématique des systèmes et de leur contrôle

Jacques-Louis LIONS (1973-1998)

Histoire de la civilisation moderne Emmanuel LE ROY LADURIE (1973-1999)

La Grèce et la formation de la pensée morale et politique

Jacqueline DE ROMILLY (1973-1984)

Théorie des groupes Jacques TITs (1973-2000)

Étude comparée des religions antiques Jean-Pierre VERNANT (1975-1984)

Histoire sociale et intellectuelle de la Chine

Jacques GERNET (1975-1992)

Physiologie du développement Alfred Jost (1974-1987)

Histoire des mentalités religieuses dans l'Occident moderne Jean DELUMEAU (1975-1994)

\section{4}

Embryologie expérimentale

Étienne WOLFF (1955-1974)

Méthodes physiques de l'astronomie

André LALLEMAND (1961-1974)

\section{5}

Épigraphie et antiquités grecques

Louis ROBERT (1939-1974)

Langue et littérature françaises du Moyen Âge Invention, technique et langage en musique Félix LECOY (1947-1974)

Assyriologie

René LABAT (1952-1974)

Analyse des faits économiques et sociaux

François PERROUX (1955-1974)

Sociologie musulmane

Henri LAOUST (1956-1975)
Sémiologie littéraire

Roland BARTHES (1976-1980)

Pierre BoulEZ (1976-1995)

Histoire de Rome

Paul-Marie VEYNE (1976-1999)

Communications cellulaires

Jean-Pierre CHANGEuX (1975-2006)

Neuropsychologie du développement Julian DE AJURIAGUERRA (1975-1981)

Histoire et civilisation du monde byzantin Gilbert DAGRON (1975-2001)

Langue et littérature arabes classiques André MIQUEL (1976-1997) 
Psychologie des arts plastiques

René HUYGHE (1951-1976)

Médecine expérimentale

Bernard HALPERN (1961-1975)

Géographie du continent européen

Maurice LELANNOU (1969-1976)

Histoire de la création artistique en France Jacques THUILLIER (1977-1998)

Médecine expérimentale

Jean DAUSSET (1977-1987)

Christianisme et gnoses dans l'Orient préislamique

Antoine GuILLAUMONT (1977-1986)

1977

Histoire et civilisations de l'Asie centrale Louis HAMBIS (1965-1977)

Sociographie de l'Asie du Sud-Est Lucien BERNOT (1978-1985)

\section{8}

Philologie et archéologie égyptiennes

Georges POSENER (1961-1978)

Égyptologie

Jean LECLANT (1979-1990)

\section{9}

Théorie des équations différentielles et fonctionnelles

Jean LERAY (1947-1978)

Langues et littératures de l'Inde

Jean Filliozat (1952-1978)

Sociologie de la civilisation moderne

Raymond ARON (1970-1978)

Chimie organique des hormones

Alain HoREAU (1956-1980)

Sémiologie littéraire

Roland BARTHES (1976-1980)

Droit international

René-Jean DuPUY (1979-1989)

Civilisation japonaise

Bernard FRANK (1979-1996)

Chimie des interactions moléculaires

Jean-Marie LEHN (1979-2010)

\section{0}

Bio-Énergétique cellulaire

Pierre Joliot (1981-2002)

Études comparées de la fonction poétique

Yves BoNNEFOY (1981-1993)

\section{1}

Littérature latine

Pierre COURCELLE (1952-1980)

Histoire et structure sociales de Paris et de la région parisienne

Louis CHEVALIER (1952-1981)

Étude du monde chinois : institutions et concepts

Rolf A. STEIN (1966-1981)

Sociologie

Pierre BOURDIEU (1981-2001)

Étude comparée des sociétés africaines

Françoise HÉRITIER (1982-1998)

Histoire de la pensée hellénistique et romaine

Pierre HADOT (1982-1991) 
1982

Histoire sociale de l'Islam contemporain Jacques BERQUE (1956-1981)

Préhistoire

André LEROI-GOURHAN (1969-1982)

Neuropsychologie du développement Julian DE AJURIAGUERRA (1975-1981)
Physique statistique Philippe NOZIÈRES (1983-2001)

Paléoanthropologie et préhistoire Yves COPPENS (1983-2005)

Neuropharmacologie Jacques GLOWINSKI (1982-2006)

\section{3}

Histoire du monde indien

Gérard FUSSMAN (1984-2011)

Antiquités nationales

Christian GoudinEAU (1984-2010)

Analyse et géométrie

Alain CONNES (1984)

Jacques PRENTKI (1964-1983)

\section{4}

La Grèce et la formation de la pensée morale Tradition et critique des textes grecs et politique

Jacqueline DE ROMILLY (1973-1984)

Étude comparée des religions antiques

Jean-Pierre VERNANT (1975-1984)

Jean IRIGOIN (1986-1992)

Grammaire et pensée allemandes

Jean-Marie ZEMB (1986-1998)

\section{5}

Histoire des systèmes de pensée

Michel FoUCAULT (1970-1984)

Épistémologie comparative

Gilles-Gaston GRANGER (1986-1991)

Art et civilisation de la Renaissance en Italie

André CHASTEL (1970-1984)

Histoire de la France contemporaine

Maurice AGULHON (1986-1997)

Langues et civilisation de l'Asie Mineure

Emmanuel LAROCHE (1973-1985)

Assyriologie

Paul GARELLI (1986-1995)

\section{6}

Physique mathématique

André LICHNEROWICZ (1952-1986)

Magnétisme nucléaire

Anatole ABRAGAM (1960-1985)

Christianisme et gnoses dans l'Orient préislamique

Antoine GUILLAUMONT (1977-1986)

Sociographie de l'Asie du Sud-Est

Lucien BERNOT (1978-1985)

Analyse économique

Edmond MALINVAUd (1987-1993)

Géodynamique

Xavier LE PICHON (1986-2008)

Théorie linguistique

Claude HAGĖGE (1988-2006)

Rhétorique et société en Europe

(XVI ${ }^{e}$-XVII ${ }^{e}$ siècles)

Marc FUMAROLI (1987-2002)

\section{7}

Physiologie du développement

Alfred JosT (1974-1987)

Embryologie cellulaire et moléculaire

Nicole LE DOUARIN (1988-2000) 
1988

Médecine expérimentale

Jean DAUSSET (1977-1987)

Astrophysique théorique

Jean-Claude PECKER (1964-1988)

Droit international

René-Jean DuPUY (1979-1989)

Création

Littérature française moderne

Georges BLIN (1965-1988)

Égyptologie

Jean LECLANT (1979-1990)

Philosophie de la connaissance Jules VuILLEMIN (1962-1990)

Histoire des sociétés médiévales Georges DUBY (1970-1991)

Neurophysiologie

Yves LAPORTE (1972-1991)

Épistémologie comparative

Gilles-Gaston GRANGER (1986-1990)

Chaire européenne

Génétique cellulaire

François JACOB (1964-1991)

Étude du Bouddhisme

André BAREAU (1971-1991)

Histoire sociale et intellectuelle de la Chine Jacques GERNET (1975-1992)

Histoire de la pensée hellénistique et romaine

Pierre HADOT (1982-1991)

Chaire européenne

Création
Médecine expérimentale

Pierre CORVOL (1989-2012)

\section{9}

Astrophysique observationnelle

Antoine LABEYRIE (1991)

Histoire des syncrétismes de la fin de l'Antiquité

Michel TARDIEU (1991-2008)

Chaire européenne

Harald WEINRICH (1989-1990)

\section{0}

Histoire de la Chine moderne

Pierre-Étienne WILL (1991)

Égyptologie

Jean YOYOTTE (1991-1997)

\section{1}

Langues et littératures romanes Harald WEINRICH (1992-1998)

Histoire de l'Occident méditerranéen au Moyen Âge

Pierre TOUBERT (1992-2003)

Physiologie de la perception et de l'action

Alain BERTHOZ (1992-2010)

Histoire et anthropologie des sociétés méso- et sud-américaines

Nathan WACHTEL (1992-2005)

Wolf LEPENIES (1991-1992)

\section{2}

Génétique moléculaire

Pierre CHAMBON (1993-2002)

Histoire moderne et contemporaine du monde russe

François-Xavier CoQUIN (1993-2001)

Langues et religions indo-iraniennes Jean KELLENS (1993)

Histoire économique et monétaire de l'Orient hellénistique

Georges LE RIDER (1993-1998)

Umberto ECO (1992-1993)

Chaire internationale

Bronislaw GEREMEK (1992-1993) 
1993

Anthropologie physique

Jacques RUFFIÉ (1972-1992)

Études comparées de la fonction poétique Yves BONNEFOY (1981-1993)

Tradition et critique des textes grecs Jean IRIGOIN (1986-1992)

Chaire européenne

Chaire internationale

Physiologie cellulaire

François MOREL (1967-1993)

Hébreu et araméen

André CAQUOT (1972-1994)

Histoire des mentalités religieuses dans l'Occident moderne

Jean DELUMEAU (1975-1994)

Analyse économique

Edmond MaLinVAUd (1987-1993)

Chaire européenne

Chaire internationale

Algèbre et géométrie

Jean-Pierre SERRE (1956-1994)

Invention, technique et langage en musique Pierre BoulEz (1976-1995)

Assyriologie

Paul GARELLI (1986-1995)

Chaire européenne

Chaire internationale

Biochimie cellulaire

François GROS (1973-1996)

Chaire européenne

Chaire internationale
Fondements et principes de la reproduction humaine

Étienne BAULIEU (1993-1998)

Littératures de la France médiévale Michel ZINK (1994)

Les civilisations de l'Europe au néolithique et à l'âge du bronze

Jean GuILAINE (1994-2007)

Werner HILDENBRAND (1993-1994)

ZHANG Guangda (1993-1994)

\section{4}

Biologie moléculaire des plantes Joseph SCHELL (1994-1998)

Antiquités sémitiques

Javier TEIXIDOR (1995-2001)

Biologie historique et évolutionnisme Armand DE RICQLÈs (1995-2010)

Philosophie du langage et de la connaissance

Jacques BOUVERESSE (1995-2010)

Norbert OHLER (1994-1995)

Orest RANUM (1994-1995)

\section{5}

Équations différentielles et systèmes dynamiques

Jean-Christophe YoccOZ (1996)

Anthropologie théâtrale Jerzy GROTOWSKI (1996-1999)

Chimie des solides

Jean RouXEL (1996-1998)

Klaus RAJEWSKI (1995-1996)

Harris MEMEL-FoTÊ (1995-1996)

\section{6}

Immunologie moléculaire

Philippe KOURILSKY (1998-2012)

Pieter Westbroek (1996-1997)

Igor MEL'ČUK (1996-1997) 
Civilisation japonaise

Bernard FRANK (1979-1996)

Égyptologie

Jean YOYOTTE (1991-1997)

Chaire européenne

Chaire internationale

Langue et littérature arabes classiques André MiQUEL (1976-1997)

Étude comparée des sociétés africaines Françoise HÉRITIER (1982-1998)

Histoire de la France comtemporaine Maurice AGULHON (1986-1997)

Langues et littératures romanes Harald WEINRICH (1992-1998)

Biologie et génétique du développement Étienne-Émile BAULIEU (1993-1998)

Chaire européenne

Chaire internationale

Analyse mathématique des systèmes et de leur contrôle

Jacques-Louis LiONS (1973-1998)

Histoire de la création artistique en France Jacques THUILLIER (1977-1998)

Grammaire et pensée allemandes Jean-Marie ZEMB (1986-1998)

Histoire économique et monétaire de l'Orient hellénistique

Georges LE RIDER (1993-1998)

Biologie moléculaire des plantes Joseph SCHELL (1994-1998)

Chimie des solides

Jean RouXEL (1996-1998)
Histoire de la France des Lumières

Daniel RocHE (1999-2005)

Histoire turque et ottomane

Gilles VEINSTEIN (1999-2012)

Abram DEsWAAN (1997-1998)

Brian STOCK (1997-1998)

\section{8}

Assyriologie

Jean-Marie DURAND (1999-2011)

Littératures modernes de l'Europe néolatine

Carlo Ossola (1999)

Théorie économique et organisation sociale Roger GUESNERIE (2000)

Histoire et civilisation du monde achéménide et de l'empire d'Alexandre Pierre BRIANT (1999-2012)

Fondements et principes de la reproduction humaine Spyros ARTAVANIS-TSAKONAS (2000-2012)

Thomas W. GaEhtgens (1998-1999)

Patrice HigONNET (1998-1999)

\section{9}

Théorie des Nombres

Don ZAGIER (2000)

Philosophie des sciences biologiques et médicales

Anne FAGOT-LARGEAUlT (2000-2009)

Philosophie et histoire des concepts scientifiques

Ian HACKING (2000-2006)

Anthropologie de la Nature Philippe DESCOLA (2000)

Physique quantique

Serge HAROCHE (2001)

Civilisation pharaonique : Archéologie, philologie, histoire

Nicolas GRIMAL (2000) 
Chaire européenne

Chaire internationale

Histoire de la civilisation moderne Emmanuel LE ROY LADURIE (1973-1999)

Histoire de Rome

Paul VEYNE (1976-1999)

Physique statistique

Philippe NOZIÈRES (1983-2001)

Embryologie cellulaire et moléculaire Nicole LE DOUARIN (1988-2000)

Anthropologie théâtrale

Jerzy GROTOWSKI (1996-1999)

Chaire européenne

Chaire internationale

Théorie des groupes

Jacques TITs (1973-2000)

Sociologie

Pierre BOURDIEU (1981-2001)

Antiquités sémitiques

Javier TEIXIDOR (1995-2001)

Chaire européenne

Chaire internationale
Hans-Wilhelm MÜLER-GÄRTNER

(1999-2000)

James Watson CRONIN (1999-2000)

\section{0}

Chimie de la matière condensée

Jacques LIVAGE (2001)

Histoire de l'art européen médiéval et moderne

Roland RECHT (2001-2012)

Évolution du climat et de l'océan

Édouard BARD (2001)

Génétique et physiologie cellulaire

Christine PETIT (2001)

Religion, institutions et société de la Rome antique

John SCHEID (2001)

Michael EDWARDS (2000-2001)

Miklós SzABÓ (2000-2001)

\section{1}

Histoire moderne et contemporaine du politique

Pierre RosANVALlon (2001)

Études juridiques comparatives et internationalisation du droit

Mireille DELMAS-MARTY (2002-2011)

Équations aux dérivées partielles et applications

Pierre-Louis LIONS (2002)

Claudio MAGRIS (2001-2002)

Paul FARMER (2001-2002)

\section{2}

Histoire et civilisation du monde byzantin Gilbert DAGRON (1975-2001)

\section{Rhétorique et sociétés en Europe} (XVI ${ }^{e}-X V I I^{e}$ siècles $)$

Marc FUMAROLI (1987-2002)

Histoire moderne et contemporaine du monde russe

François-Xavier COQUIN (1993-2001)

Génétique moléculaire

Pierre CHAMBON (1993-2002)
Étude de la création littéraire en langue anglaise

Michael EDWARDS (2003-2008)

Épigraphie et histoire des cités grecques Denis KNOEPFLER (2003)

Histoire contemporaine du monde arabe Henry LAURENS (2003)

Génétique humaine

Jean-Louis MANDEL (2003) 
Chaire européenne

Chaire internationale

Bioénergétique cellulaire

Pierre JoLIOT (1981-2002)

Chaire européenne

Chaire internationale

Physique atomique et moléculaire

Claude COHEN-TANNOUDJI (1973-2004)

Histoire de l'Occident méditerranéen au Moyen-Âge

Pierre TOUBERT (1992-2003)

Chaire européenne

Chaire internationale

Physique de la matière condensée

Pierre-Gilles DE GENNES (1971-2004)

Physique corpusculaire

Marcel FROISSART (1973-2004)

Histoire de la France des Lumières

Daniel RocHE (1999-2005)

Chaire européenne

Chaire internationale

Chaire de création artistique

Histoire et anthropologie des sociétés méso-et sud-américaines

Nathan WACHTEL (1992-2005)

Chaire européenne

Chaire internationale

Chaire de création artistique

Chaire d'innovation technologiqueLiliane Bettencourt
Hans BeLtiNG (2002-2003)

Stuart EDELSTEIN (2002-2003)

\section{3}

Particules élémentaires, gravitation et cosmologie

Gabriele VENEZIANO (2004)

Theodor BERCHEM (2003-2004)

Jayant Vishnu NARLIKAR (2003-2004)

\section{4}

Rationalité et sciences sociales Jon ELSTER (2006-2011)

Psychologie cognitive expérimentale Stanislas DEHAENE (2005)

Sandro STRINGARI (2004-2005)

Celâl SENGÖR (2004-2005)

\section{5}

Physique mésoscopique

Michel DeVoret (2007)

Littérature française moderne et contemporaine : histoire, critique, théorie

Antoine COMPAGNON (2006)

Écrit et cultures dans l'Europe moderne

Roger CHARTIER (2006)

Maurice BLOCH (2005-2006)

Thomas PAVEL (2005-2006)

Christian DE PORTZAMPARC (2005-2006)

\section{6}

Processus morphogénétiques

Alain PROCHIANTZ (2007)

Daniele ViTALI (2006-2007)

Guy ORBAN (2006-2007)

Pascal DUSAPIN (2006-2007)

Jean-Paul CLOZEL (2006-2007) 
Communications cellulaires

Jean-Pierre CHANGEUX (1975-2006)

Neuropharmacologie

Jacques GLOWINSKI (1982-2006)

Paléoanthropologie et préhistoire

Yves COPPENS (1983-2005)

Théorie linguistique

Claude HAGÈGE (1988-2006)

Les civilisations de l'Europe au néolithique et à l'âge du bronze

Jean GUILAINE (1994-2007)

Philosophie et histoire des concepts scientifiques

Ian HACKING (2000-2006)

Chaire européenne

Chaire internationale

Chaire de création artistique

Chaire d'innovation technologiqueLiliane Bettencourt
Chimie des processus biologiques

Marc FONTECAVE (2008)

Microbiologie et maladies infectieuses

Philippe SANSONETTI (2007)

Paléontologie humaine

Michel BRUNET (2007-2011)

Milieux bibliques

Thomas RÖMER (2007)

Physique de la matière condensée

Antoine GEORGES (2009)

Histoire intellectuelle de la Chine Anne CHENG (2008)

Manfred KROPP (2007-2008)

Pierre MAgistRetTI (2007-2008)

Ariane MNOUCHKINE (2007-2008), non occupée

Gérard BERRY (2007-2008)

\section{8}

Chaire européenne - Développpement durable Henri LERIDON (2008-2009)

Chaire internationale - Savoirs contre pauvreté Esther DuFLo (2008-2009)

Chaire de création artistique

Pierre-Laurent AIMARD (2008-2009)

Chaire d'innovation technologique-

Mathias FINK (2008-2009) Liliane Bettencourt

Géodynamique

Xavier LE PICHON (1986-2008)

Études de la création littéraire en langue anglaise

Michael EDWARDS (2003-2008)

Chaire de création artistique

Chaire Développement durable Environnement, énergie et société

Chaire Savoirs contre pauvreté

Chaire d'Informatique et sciences numériques

Chaire d'Innovation technologique Liliane Bettencourt
Chimie des matériaux hybrides

Clément SANCHEZ (2011)

Métaphysique et philosophie de la connaissance

Claudine TIERCELIN (2010)

Jacques NICHET (2009-2010)

Nicolas STERN (2009-2010)

Peter PIOT (2009-2010)

Gérard BERRY (2009-2010)

Patrick Couvreur (2009-2010) 
2010

Histoire des syncrétismes de la fin de l'Antiquité

Michel TARDIEU (1991-2008)

Philosophie des sciences biologiques et médicales

Anne Fagot-Largeault (2000-2009)

Chimie de la matière condensée

Jacques LIVAGE (2001-2009)

Chaire de création artistique

Chaire Développement durable -

Environnement, énergie et société

Chaire Savoirs contre pauvreté

Techniques et économies

de la Méditerranée antique

Jean-Pierre BRUN (2011)

Philologie de la civilisation japonaise Jean-Noël ROBERT (2011)

Physique de l'intérieur de la terre

Barbara ROMANOWICZ (2011)

Anselm KIEFER (2010-2011)

Jean-Marie TARASCON (2010-2011)

Ismail SERAGELDIN (2010-2011)

Chaire d'Informatique et sciences numériques Martin ABADI (2010-2011)

Chaire d'Innovation technologique

Elias ZERHOUNI (2010-2011)

Liliane Bettencourt

\section{1}

Antiquités nationales

Christian GOUDINEAU (1984-2010)

Chimie des interactions moléculaires

Jean-Marie LEHN (1989-2010)

Physiologie de la perception et de l'action

Alain BERTHOZ (1992-2010)

Philosophie du language et de la connaissance

Jacques BoUVERESSE (1995-2010)

Chaire de création artistique

Chaire Développement durable -

Environnement, énergie et société

Chaire Savoirs contre pauvreté

Chaire d'Informatique et sciences numériques

Chaire d'Innovation technologique Liliane Bettencourt
Atomes et rayonnement Jean DALIBARD (2012)

Épigénétique et mémoire cellulaire Edith HEARD (2012)

État social et mondialisation Alain SUPIOT (2012)

Algorithmes, machines et langages Gérard BERRY (2012)

Gilles CLÉMENT (2011-2012)

Paul ColONNA (2011-2012)

Manuela CARNEIRO DA CUNHA (2011-2012)

Serge ABiteboul (2011-2012)

Jean-Paul LAUMOND (2011-2012)

\section{2}

Histoire du monde indien Gérard FUSSMANN (1985-2011)

Biologie historique et évolutionnisme Armand DE RICQLÈS (1996-2010)

Assyriologie

Jean-Marie DURAND (1999-2011)
Histoire de la philosophie médiévale

Sociologie du travail créateur

Histoire et cultures de l'Asie centrale préislamique 
Rationalité et sciences sociales Jon ELSTER (2006-2011)

Paléontologie humaine Michel BRUNET (2007-2011)

Chaire de création artistique

Chaire Développement durable -

Environnement, énergie et société

Chaire Savoirs contre pauvreté

Chaire d'Informatique et sciences numériques
Chimie du solide et énergie

Histoire globale de la première modernité

Karol BEFFA (2012-2013)

Annie CAZENAve (2012-2013)

Dominique KeROUEDAN (2012-2013)

Bernard CHAZELlE (2012-2013) 